\title{
Study Of Prevalence Of Pulmonary Hypertension In Chronic Kidney Disease And Its Co-Relation With Clinical \& Biochemical Parameters.
}

\author{
Dr.Neelam Redkar ${ }^{1}$, Dr.Nitin Sarate ${ }^{2}$ \\ 1.Professor \& Head, of Department of Medicine, Dr.R.N.Cooper Hospital, Mumbai. \\ 2. Assistant Professor, Department of Medicine, Seth GSMC \& KEM Hospital, Mumbai. \\ Address for correspondence: Dr.Nitin Sarate, Assistant Professor, Department of Medicine, $2^{\text {Nd }}$ floor, Seth \\ GSMC \& KEM Hospital, Acharya Donde Road, Parel, Mumbai-400012, Maharashtra ,India.
}

\begin{abstract}
OBJECTIVES: The objectives of this prospective observational study were to study (i) the prevalence of pulmonary hypertension $(\mathrm{PH})$ in patients who are on conservative management, haemodialysis or continuous ambulatory peritoneal dialysis, (ii) the co-relation of $\mathrm{PH}$ with variables such as A-V fistula, hypertension, diabetes Mellitus \& duration for dialysis, and (iii) the co-relation of $\mathrm{PH}$ with biochemical parameters.MATERIALS AND METHODS: The study was conducted in a tertiary care centre in Mumbai over a period 12 months after obtaining approval from the Institutional Ethics Committee. 245 CKD patients, on conservative management, haemodialysis and continuous ambulatory peritoneal dialysis were enrolled in this study, taking into consideration the inclusion \& exclusion criteria and appropriate informed consent after taking detailed history of every patient. All patients underwent routine investigations and 2D ECHO for pulmonary hypertension. RESULTS: Our study enrolled 245 patients in the age group of 20 to 85 years where $68.6 \%$ patients were male and 31.4\% were females. 28.2\% patients had CKD with PH while $71.8 \%$ had CKD without $\mathrm{PH}$. The proportion of patients with PH having AV fistula was significantly more than those without AV fistula. Of the $28.2 \%$ patients with $\mathrm{PH}, 33.6 \%$ had hypertension and $22 \%$ were diabetic. Our study revealed a positive association between the duration of dialysis and the prevalence of PH along-with low levels of haemoglobin and serum bicarbonate, and high levels of uric acid, BUN and creatinine.

CONCLUSION: The prevalence of $\mathrm{PH}$ was found to be $28.2 \%$. PH is positively correlated with chemical as well as biochemical parameters.
\end{abstract}

Keywords: A-V fistula, CKD with PH, hemodialysis, Prevalence study, pulmonary arterial pressure.

\section{INTRODUCTION}

Cardiovascular disease is a well-known and significant source of mortality in patients with chronic kidney disease $(\mathrm{CKD}){ }^{(1,2,3)}$ inspite of the latest advances in the care of patients undergoing renal replacement treatment. $^{(4)}$

Pulmonary hypertension $(\mathrm{PH})$ is an overlooked cardiovascular morbidity in patients of chronic kidney disease who are on conservative management and haemodialysis. As pulmonary hypertension is a hemodynamic state defined by a resting mean pulmonary artery pressure at or above $25 \mathrm{~mm} \mathrm{Hg},{ }^{(5)}$ it can be the result of a variety of diseases of different causes.

Pulmonary hypertension is an elevation of pulmonary arterial pressure (PAP) that can be the result of heart, lung or systemic disorders. ${ }^{(6,7)} \mathrm{PH}$ is an increase in blood pressure in the lung vasculature, resulting in symptoms like shortness of breath, dizziness, fainting etc. that are exacerbated by exertion and can be a severe disease with a markedly decreased exercise tolerance and lead to heart failure.

The evolution of PH in CKD frequently originates with the interaction of a predisposing state and one or more inciting stimuli, a concept referred to as the "multiple-hit hypothesis." (8,9)

$\mathrm{PH}$ has been commonly found in patients undergoing renal replacement with haemodialysis via an arterio-venous graft or fistula, ${ }^{(6,7)}$ and is characterized by elevations in the pulmonary arterial pressure and pulmonary vascular resistance (PVR) that eventually result in right ventricular failure and even premature death. Chronic renal failure (CRF) is most often seen with patients with PH. ${ }^{(10)}$ Chronic haemodialysis patients are continuously exposed to multifactorial pulmonary insults. Recent studies have demonstrated a $40 \%$ incidence of $\mathrm{PH}$ on detection by Doppler echocardiography (ECG) in patients with end-stage renal disease (ESRD) on chronic haemodialysis (HD) therapy via arterial-venous (A-V) access.$^{(11)}$ A study done by C. J. Rhodes et al has proved the role of iron in the natural history of pulmonary hypertension. High hepcidin levels underlie the anaemia of chronic disease, like CKD. ${ }^{(12)}$

Uric acid levels are elevated in patients with PH and correlate with haemodynamics, which was also shown by a study done by Norotoshi Nagaya et al in which serum UA increased in proportion to the clinical severity of 
Primary Pulmonary Hypertension(PPH) and found to have an independent association with long-term mortality of patients with PPH. ${ }^{(13)}$ This study was conducted to study to see the profile of patients of PH in CKD that are only on medical management and on renal replacement therapy.

\section{OBJECTIVES}

The primary objective of this prospective observational study was to study the prevalence of PH in patients who are on conservative management, haemodialysis or continuous ambulatory peritoneal dialysis.

The secondary objective was to study the co-relation of $\mathrm{PH}$ with variables such as A-V fistula, hypertension, diabetes mellitus \& duration for dialysis, and it's co-relation with biochemical parameters.

\section{MATERIALS AND METHODS}

This study was conducted in a tertiary care centre in Mumbai over 12 months after obtaining approval from the Institutional Ethics Committee. 245 CKD patients that are on conservative management, haemodialysis and continuous ambulatory peritoneal dialysis were enrolled in this study based on the inclusion \& exclusion criteria.

Once all the criteria were satisfied, a written informed consent was taken and the patient was included in the study.A detailed history of every patient including age, sex, duration of illness, etiology of chronic kidney disease, type of treatment received: either conservative, haemodialysis or continuous ambulatory peritoneal dialysis (CAPD) and its duration were noted.Each patient underwent routine investigations like complete haemogram, serum electrolytes $\left(\mathrm{Na}, \mathrm{K}, \mathrm{Ca}, \mathrm{PO}_{4}, \mathrm{UA}\right)$, renal function tests (BUN, serum creatinine), liver function tests (Total /direct bilirubin, AST, ALT, ALP), urine routine \& microscopy and some specific investigations like arterial blood gas (ABG), Chest X-Ray, ECG, USG abdomen, autoimmune work up like ANA (as and when indicated).

Each patient underwent 2D echocardiography for $\mathrm{PH}$.

3.1 Inclusion criteria

All patients $>12$ years of age with chronic renal failure either on conservative management, haemodialysis or continuous ambulatory peritoneal dialysis were included.

3.2 Exclusion criteria

Patients under the age of 12 years, those with a history of smoking, primary PH \& PH secondary to either causes and pregnant \& postpartum females were excluded from the study.

The results were statistically analysed, compared with previous studies and conclusions derived appropriately.

\section{RESULTS}

Our study enrolled patients in the age group of 20 to 85 years where $68.6 \%$ patients were male and $31.4 \%$ were females. 245 patients underwent 2D echocardiography performed by cardiologist of the tertiary institute. Pulmonary hypertension as defined previously ${ }^{14}$ was found in 69 cases.

28.2\% patients were found to have CKD with PH while the rest $71.8 \%$ had CKD without PH. 30.0\% patients had $\mathrm{PH}$ and were under maintenance treatment as compared to $24.7 \%$ cases who were on conservative treatment. Our study identified $15.9 \%$ patients with mild $\mathrm{PH}$ followed by $9.8 \%$ patients with moderate $\mathrm{PH}$ and $2.5 \%$ patients with severe $\mathrm{PH}$, as depicted in the below "Figure 1".

Figure 1:

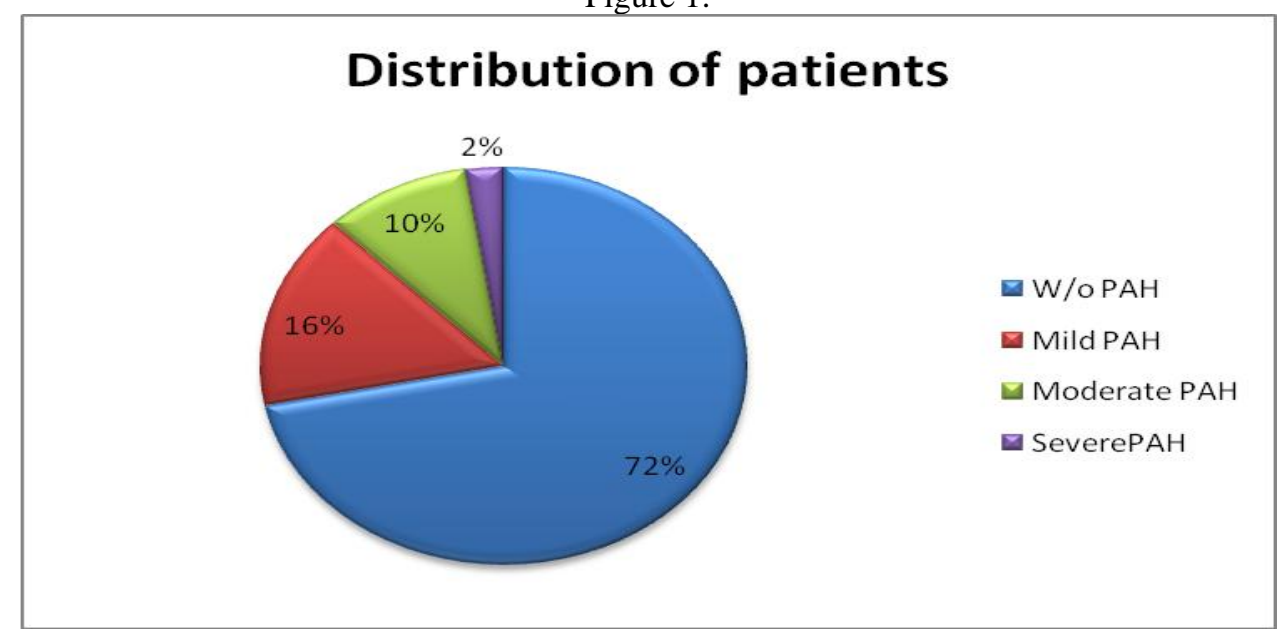

Table 1: Prevalence of PH in Chronic Kidney Disease in study group 


\begin{tabular}{|c|c|c|c|}
\hline $\begin{array}{c}\text { Treatment } \\
\text { modality }\end{array}$ & $\begin{array}{c}\text { No. of CKD } \\
\text { patients }\end{array}$ & No. of CKD with PH & Prevalence (\%) \\
\hline $\begin{array}{c}\text { Group 1 } \\
\text { Maintenance HD }\end{array}$ & 160 & 48 & 30 \\
\hline $\begin{array}{c}\text { Group 2 } \\
\text { Conservative }\end{array}$ & 85 & 21 & 24.7 \\
\hline
\end{tabular}

The data represented in the above "TABLE 1", shows that $30.0 \%$ patients with PH were on maintenance HD which was more when compared to $24.7 \%$ patients that were on conservative treatment, though the difference was not found to be statistically significant.

The proportion of patients with PH having AV Fistula was significantly more (42.2\%) when compared to those without AV Fistula (25\%).

Of the $28.2 \%$ patients with $\mathrm{PH}, 33.6 \%$ had hypertension and $22 \%$ were diabetic.

The association between the duration of dialysis and $\mathrm{PH}$, after applying the Chi-square test, is graphically represented in "Fig. 2".

Figure 2: Association between duration of dialysis and PH

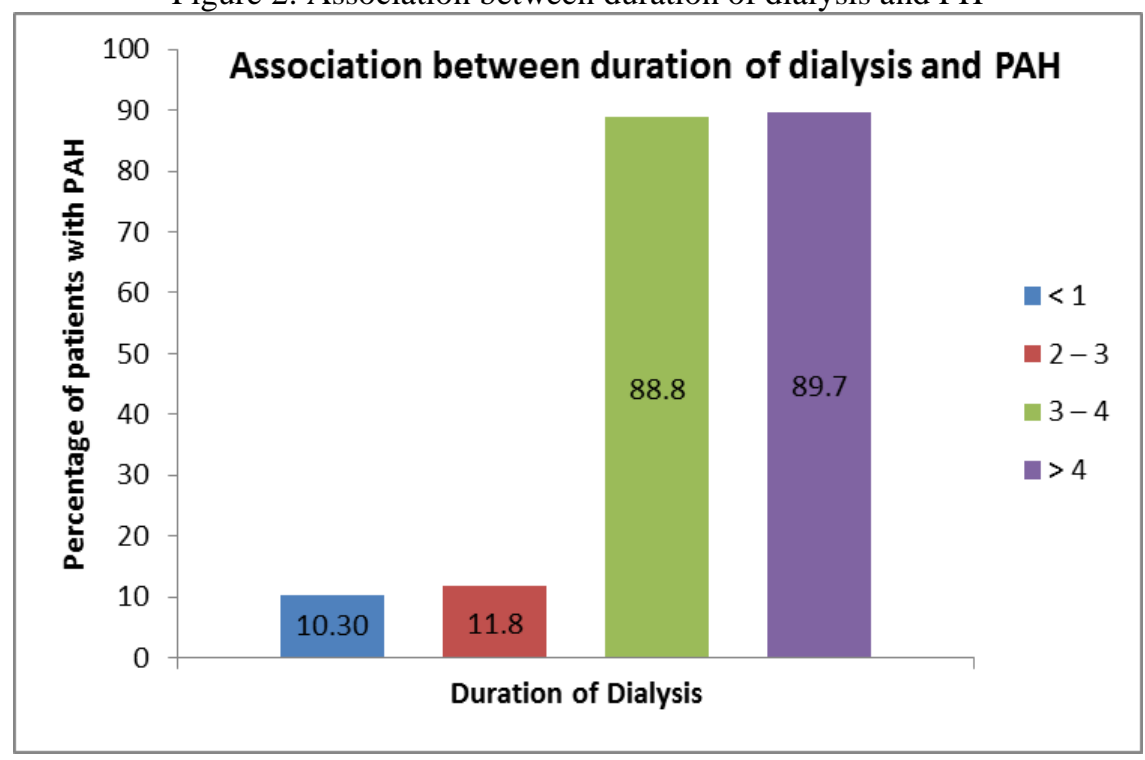

Table 2: Biochemical parameters

\begin{tabular}{|l|l|l|l|l|}
\hline $\begin{array}{l}\text { Sr. } \\
\text { No. }\end{array}$ & Biochemical parameters (units) & $\begin{array}{l}\text { No. of PH } \\
(\mathbf{Y} / \mathbf{N})\end{array}$ & $\begin{array}{l}\text { Mean value } \\
(\overline{\mathbf{X}} \pm \mathbf{S D})\end{array}$ & P value \\
\hline 1 & Haemoglobin $(\mathrm{g} \%)$ & Yes -69 & $7.91 \pm 1.21$ & $* 0.034$ \\
\hline & & No -176 & $8.53 \pm 1.31$ & \\
\hline 2 & Uric Acid $(\mathrm{mg} \%)$ & Yes -69 & $6.44 \pm 1.76$ & $* 0.012$ \\
\hline & & No -176 & $5.95 \pm 1.44$ & \\
\hline 3 & Serum Bicarbonate $(\mathrm{mmol} / \mathrm{L})$ & Yes -69 & 8.6802 .01 & $* 0.029$ \\
\hline & & No -176 & $11.06 \pm 01.94$ & \\
\hline 4 & BUN (mg\%) & Yes -69 & $75.48 \pm 30.20$ & $* 0.042$ \\
\hline & & No -176 & $66.34 \pm 32.17$ & \\
\hline 5 & Serum Creatinine $(\mathrm{mg} \%)$ & Yes -69 & 08.8303 .01 & $* 0.036$ \\
\hline & \multicolumn{5}{|l|}{ No -176} & 07.9403 .40 & \\
\hline
\end{tabular}


The prevalence of $\mathrm{PH}$ in the haemodialysis group is $30 \%$ and that in the conservative management group is $24.7 \%$, which indicates the role of renal replacement treatment in the pathogenesis of $\mathrm{PH}$. The prevalence of $\mathrm{PH}$ in CKD in our study group was $28.2 \%$, which is comparable to $26.74 \%$ from a previous study by F. Tarrass et al, ${ }^{(15)}$ which is comparable to another previous study done by Amin M et al ${ }^{(16)}$ whose study demonstrated that $29 \%$ of patients with CRF receiving regular haemodialysis have $\mathrm{PH}$.

All CKD patients underwent 2D echocardiography. Based upon the tricuspid regurgitation velocity, systolic pulmonary arterial pressure can be estimated according to the following equation: Systolic PAP $=4$ (TR)+ RAP. PH in CKD patients is graded by the reporting cardiologist as mild $(35-45 \mathrm{mmHg})$, moderate $(45-60 \mathrm{mmHg})$ or severe $(>60 \mathrm{mmHg}){ }^{(17)}$ In this study, out of 245 patients, we found $\mathrm{PH}$ according to echographic criteria in 69 patients. Patients were graded according to pulmonary artery systolic pressure. We found 39 patients have mild $\mathrm{PH}, 24$ have moderate $\mathrm{PH}$ and 6 patients have severe $\mathrm{PH}$.

The presence of $42.2 \%$ patients having AV Fistula for haemodialysis in patients with CKD along-with $\mathrm{PH}$ are comparable with two previous studies by Yingla et al ${ }^{(11)}$ and Abassi Zaid et al. ${ }^{(18)}$

There are no previous studies that show the prevalence of $\mathrm{PH}$ in hypertensive and diabetic patients.

Our study revealed a positive association between the duration of dialysis and the prevalence of PH. A similar study by Patel P et al ${ }^{(19)}$ showed that as duration of renal failure increased so did the chance of developing PH. PH is positively correlated with clinical parameters like RRT-haemodialysis, A-V fistula, duration of dialysis and biochemical parameters like low haemoglobin, low sr. bicarbonate, high levels of uric acid, BUN, creatinine.

Our study result demonstrated high mean Haemoglobin indicating a role of anaemia in the pathogenesis of PH in CKD. This co-relation is supported by C. J. Rhodes et al ${ }^{(12)}$ showing the role of iron in the natural history of PH. Iron availability influences the pulmonary vasoconstrictor response to hypoxia and accumulating evidence indicates that iron deficiency is prevalent in idiopathic and heritable forms of $\mathrm{PH}$.

The mean serum uric acid level found in our study is supported by Norotoshi Nagaya et al ${ }^{(13)}$ showing that serum UA increases in proportion to the clinical severity of PH and has independent association with long-term mortality of patients with Primary PH indicating it as a predictor of morbidity \& mortality in PH.

The findings of mean serum bicarbonate levels are comparable to the study done by Patel et al ${ }^{(19)}$ and indicate a positive correlation between $\mathrm{PH}$ and low bicarbonate level, leading to metabolic acidosis. The results of BUN \& serum creatinine levels in our study are comparable to that of Patel et al ${ }^{19}$ indicating PH in CKD and correlates with high levels of both.

\section{CONCLUSION}

In this prospective study, prevalence of pulmonary aterial hypertension(PAH) in chronic kidney disease is $28.2 \%$. The prevalence of pulmonary arterial Hypertension[PAH] in hemodialysis group i.e. Group 1 is $30 \%$ and in conservative management group i.e Group 2 is $24.7 \%$, indicating the role of renal replacement treatment in the pathogenesis of pulmonary hypertension. Pulmonary hypertension in CKD group having A-V fistula is $42.2 \%$, indicating role of A-V fistula in the pathogenesis of PAH. Though PAH is found more in previous hypertensive patients but is insignificant statistically, same with the case of diabetes mellitus. Duration of dialysis is important factor in the pathogenesis of PAH, indicated by $88.8 \%$ of cases with PAH has $3-4$ years of dialysis and $89.7 \%$ with PAH has $>4$ years of dialysis which is significantly more as compared to $11.8 \%$ with $2-3$ yrs of dialysis and $10.3 \%$ with $<1$ year of dialysis.

Thus in summary, prevalence of pulmonary hypertension in chronic kidney disease is $28.2 \%$. Pulmonary hypertension is positively correlated with clinical parameters like RRT-hemodialysis, A-V fistula, duration of dialysis and biochemical parameters like low haemoglobin, low sr. bicarbonate, high levels of uric acid, BUN, creatine. 6] $\mathrm{PAH}$ is also correlated positively with biochemical parameters like anaemia(low $\mathrm{Hb} \%$ ), low bicarbonate level, high serum uric acid, high blood urea nitrogen(BUN), high serum creatine level.

\section{REFERENCES}

[1] Drey N, Roderick P, Mullee M, Rogerson M. A population-based study of the incidence and outcomes of diagnosed chronic kidney disease. Am J Kidney Dis. 2003 Oct;42(4):677-84.

[2] Muntner P, He J, Hamm L, Loria C, Whelton PK. Renal insufficiency and subsequent death resulting from cardiovascular disease in the United States. J Am Soc Nephrol. 2002 Mar;13(3):745-53.

[3] Shlipak MG, Stehman-Breen C, Vittinghoff E, Lin F, Varosy PD, Wenger NK, Furberg CD. Creatinine levels and cardiovascular events in women with heart disease: do small changes matter? Am J Kidney Dis. 2004 Jan;43(1):37-44.

[4] Collins AJ, Kasiske B, Herzog C, et al. Excerpts from the United States Renal Data System 2006 Annual Data Report. Am J Kidney Dis. 2007 Jan;49(1 Suppl 1):A6-7, S1-296. 
[5] Badesch DB, Champion HC, Sanchez MA, et al. Diagnosis and assessment of pulmonary arterial hypertension. J Am Coll Cardiol. 2009 Jun 30;54(1 Suppl):S55-66. doi: 10.1016/j.jacc.2009.04.011. Review.

[6] Archer S, Rich S et al. Primary pulmonary hypertension: a vascular biology and translational research 'work in progress'. Circulation 2000; 102: 2781-2791.

[7] Yigla M, Dabbah S, Azzam ZS, Rubin AH, Reisner SA. Background diseases in 671 patients with moderate to severe pulmonary hypertension. Isr Med Assoc J. 2000 Sep;2(9):684-9.

[8] Yuan JX, Rubin LJ. Pathogenesis of pulmonary arterial hypertension: the need for multiple hits. Circulation. 2005 Feb 8;111(5):534-8.

[9] Machado RD, James V, Southwood M, et a 1. Investigation of second genetic hits at the BMPR2 locus as a modulator of disease progression in familial pulmonary arterial hypertension. Circulation.2005; 111: 607-613.

[10] Yigla M, Azzam Z, Rubin AHE, et al: Background disease in 714 patients with moderate to severe pulmonary hypertension. Med Assoc J 2000; 2:501-506.

[11] Yigla M, Nakhoul F, Sabag A, et al. Pulmonary hypertension in patients with end-stage renal disease. Chest. 2003 May;123(5):1577-82.

[12] Rhodes CJ, Wharton J, Howard L, Gibbs JS, Vonk-Noordegraaf A, Wilkins MR. Iron deficiency in pulmonary arterial hypertension: a potential therapeutic target. Eur Respir J. 2011 Dec;38(6):1453-60.

[13] Nagaya N, Uematsu M, Satoh T, et al. Serum uric acid levels correlate with the severity and the mortality of primary pulmonary hypertension. Am J Respir Crit Care Med. 1999 Aug;160(2):487-92.

[14] Clarkson MR, Giblin L, Brown A, Little D, Donohoe J. Reversal of pulmonary hypertension after ligation of a brachiocephalic arteriovenous fistula. Am J Kidney Dis. 2002 Sep;40(3):E8.

[15] F Tarrass, M Benjelloun, K Hachim, MG Benghanem, B Ramdani. Pulmonary hypertension in patients with end-stage renal disease. Indian J Nephrol. 2005;15:223-226.

[16] Amin M, Fawzy A, Hamid MA, Elhendy A. Pulmonary hypertension in patients with chronic renal failure: role of parathyroid hormone and pulmonary artery calcifications. Chest. 2003 Dec;124(6):2093-

[17] Schiller NB. Pulmonary artery pressure estimation by Doppler and two-dimensional echocardiography. Cardiol Clin. 1990 May;8(2):277-87. Review.

[18] Abassi Z, Nakhoul F, Khankin E, Reisner SA, Yigla M. Pulmonary hypertension in chronic dialysis patients with arteriovenous fistula: pathogenesis and therapeutic prospective. Curr Opin Nephrol Hypertens. 2006 Jul;15(4):353-60. Review.

[19] Patel P. et al. Clinical and biochemical parameter in chronic kidney disease. Indian journal of nephrology . Jan2007; (17): Issue 1. [IP: 202.177.228.90] 